\title{
RNAi-mediated Heterochromatin Assembly in Fission Yeast
}

\author{
M. ZoFALL AND S.I.S. GREWAL \\ Laboratory of Molecular Cell Biology, National Cancer Institute, National Institutes of Health, \\ Bethesda, Maryland 20892
}

\begin{abstract}
The organization of DNA into heterochromatin domains is critical for a variety of chromosomal functions, including gene silencing, recombination suppression, and chromosome segregation. In fission yeast, factors involved in the RNAi pathway such as Argonaute, Dicer, and RNA-dependent RNA polymerase are required for assembly of heterochromatin structures. The RNAi Argonaute-containing RITS complex and RNA-dependent RNA polymerase localize throughout heterochromatin domains. These factors are important components of a self-reinforcing loop mechanism operating in cis to process repeat transcripts into siRNAs, which involve in heterochromatin assembly. In this paper, we describe our results suggesting that slicing of repeat transcripts by the Argonaute is an important step in their conversion into siRNAs and heterochromatic silencing. Mutations in conserved residues known to be essential for slicer activity of Argonautes result in loss of siRNAs corresponding to centromeric repeats, accumulation of repeat transcripts, and defects in heterochromatin assembly. We also discuss our recent finding that heterochromatin proteins such as Swi6/HP1 serve as a platform that could recruit both silencing and antisilencing factors to heterochromatic loci.
\end{abstract}

Eukaryotic genomes contain large tracts of repetitive DNA sequences that are often packaged in the form of condensed, inaccessible heterochromatic structures (Hall and Grewal 2003). The assembly of heterochromatic structures at different loci regulates a variety of chromosomal processes. In addition to transcriptional repression, heterochromatin prohibits recombination throughout large chromosomal domains. These repressive effects of heterochromatin are widely believed to reflect the need for organisms to prohibit the transcription and proliferation of transposable DNA elements, which, due to their ability to transpose or recombine with other elements, are a major cause of genomic instability (Birchler et al. 2000; Henikoff 2000; Hall and Grewal 2003). Heterochromatin has also been shown to play an important role in regulating gene expression, ensuring proper segregation of chromosomes, and facilitating long-range chromatin interactions between distant chromosomal regions (Bernard et al. 2001; Nonaka et al. 2002; Jia et al. 2004b; Pidoux and Allshire 2004). How heterochromatin mediates diverse cellular functions is the focus of intense research in different systems.

The study of heterochromatin assembly and its various functions is particularly amenable to genetic and biochemical analyses in the model organism fission yeast Schizosaccharomyces pombe. Several trans-acting factors involved in heterochromatin formation in fission yeast are conserved in higher eukaryotic species (Grewal and Elgin 2002; Cam and Grewal 2004), and recent studies strongly suggest that similar mechanisms of heterochromatin assembly might operate in fission yeast and mammals (Grewal and Elgin 2002; Hall and Grewal 2003; Hiragami and Festenstein 2005; Ebert et al. 2006; Huisinga et al. 2006). In particular, an evolutionarily conserved Clr4 protein - the fission yeast homolog of mammalian Suv39hhas been shown to specifically methylate lysine 9 of histone H3 (H3K9), which serves as a binding site for recruitment of chromodomain proteins, including Swi6, a structural and functional homolog of mammalian hete- rochromatin protein 1 (HP1), to heterochromatic loci (Lorentz et al. 1992; Ivanova et al. 1998; Rea et al. 2000; Thon and Verhein-Hansen 2000; Bannister et al. 2001; Nakayama et al. 2001; Sadaie et al. 2004). Recent work has led to the surprising discovery of a critical role of the RNA interference (RNAi) pathway in the nucleation and assembly of heterochromatic structures at specific loci of the fission yeast genome (Hall et al. 2002; Volpe et al. 2002). Significant progress has been made in elucidating the role of small RNAs and the RNAi machinery in the assembly of heterochromatic structures (Motamedi et al. 2004; Noma et al. 2004; Verdel et al. 2004; Matzke and Birchler 2005; Sugiyama et al. 2005). These studies suggest that the RNAi machinery operates as a stable component of heterochromatin domains to promote both transcriptional and posttranscriptional silencing of heterochromatic sequences. In this paper, we describe our recent progress in understanding the mechanism of heterochromatin assembly and silencing in fission yeast.

\section{HETEROCHROMATIC REGIONS IN THE FISSION YEAST GENOME}

Fission yeast chromosomes contain large blocks of heterochromatin associated with a variety of repeat elements, in addition to small heterochromatic "islands" associated with a few meiosis-specific genes (Fig. 1) (Hall and Grewal 2003; Cam et al. 2005). Mapping of heterochromatin markers such as H3K9 methylation and Swi6 has identified extended chromosomal domains coated with heterochromatin complexes at centromeres, telomeres, and the mating-type locus (Partridge et al. 2000; Cam et al. 2005). All three heterochromatic regions share a common featureeach of these domains contains $d g / d h$ repeat elements that are preferential targets of RNAi-mediated heterochromatin formation (Chikashige et al. 1989; Hahnenberger et al. 1991; Grewal and Klar 1997; Hall et al. 2002; Volpe et al. 2002; Cam and Grewal 2004; Kanoh et al. 2005). 
a

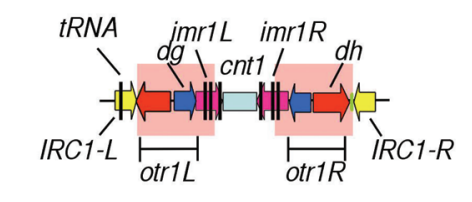

cen1

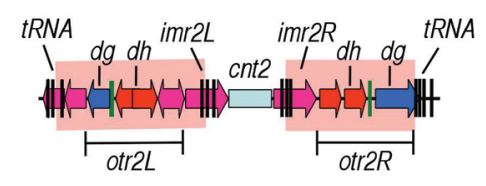

cen2

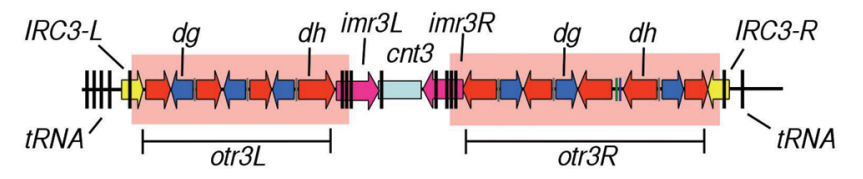

\section{cen3}

b

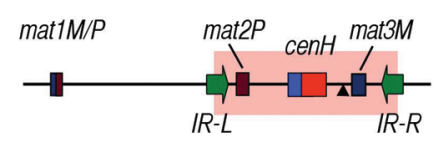

C

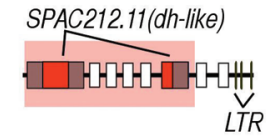

d

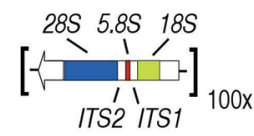

\section{Mating-type locus}

Left subtelomere 1

rDNA

Figure 1. Heterochromatin domains in the fission yeast genome. Schematic diagrams of the major heterochromatic regions in the fission yeast genome, such as centromeres, telomeres, mating-type region, and $r D N A$ locus, are shown. The regions coated with heterochromatin markers (such as $\mathrm{H} 3 \mathrm{~K} 9 \mathrm{me}$ and Swi6) and RNAi machinery are shaded in pink. (a) Each centromere contains a unique central core $(\mathrm{cnt})$, flanked by innermost inverted repeat $(\mathrm{imr})$ and outer repeat region (otr), composed of a complex arrangement of tan$\operatorname{dem} d g$ and $d h$ repeats. Clusters of $t R N A$ genes and/or $I R C$ inverted repeats surround heterochromatin domains at centromeres. (b) At the mating-type region, mat 2 and mat 3 genes are located within a $20-\mathrm{kb}$ heterochromatin domain exhibiting both suppression of transcription and recombination. A cen $H$ element that shares strong homology with $d g$ and $d h$ centromeric repeats serves as an RNAidependent heterochromatin nucleation center. Heterochromatin can also be nucleated independently at a site between cen $H$ and $m a t 3$ (black triangle) that contains binding sites for Atf1/Pcr1. The heterochromatic domain is restricted by boundary elements $I R-L$ and $I R-$ $R$. (c) cenH-like elements (SPAC212.11) are also present at subtelomeric regions which display a broad distribution of heterochromatin and RNAi machinery. (d) Heterochromatin and RNAi factors also associate with an $r D N A$ repeat, although the mechanism of heterochromatin assembly at these loci is not well understood. Small heterochromatic "islands" are also associated with certain meiosis-specific genes such as mei4 and ssm4 (not shown).

At centromeres, which in fission yeast are large complex structures ranging in size from $35 \mathrm{~kb}$ to $110 \mathrm{~kb}$, tandem arrays of $d g / d h$ repeat elements are important constituents of pericentromeric regions that surround the central core (cnt) domain (Clarke et al. 1986; Chikashige et al. 1989; Hahnenberger et al. 1991). Heterochromatin assembled at pericentromeric repeat sequences has been shown to be critical for preferential recruitment of cohesin, which is essential for proper segregation of chromosomes during cell division (Bernard et al. 2001; Nonaka et al. 2002). Distinct trans-acting factors interact with the $c n t$ domain that primarily is enriched for factors essential for kinetochore assembly such as histone H3 variant CENP-A and Mis6 protein (Saitoh et al. 1997; Partridge et al. 2000; Takahashi et al. 2000; Bjerling and Ekwall 2002). In addition to CENP-A, low levels of histone H3 methylated at lysine $4(\mathrm{H} 3 \mathrm{~K} 4 \mathrm{me})$ are also present at the cnt domain (Cam et al. 2005), although the biological significance of $\mathrm{H} 3 \mathrm{~K} 4 \mathrm{me}$ present at this site is not known.

A copy of a $d g / d h$-like element referred to as $c e n H$ is also present at the silent mating-type region (Grewal and Klar 1997). cenH is essential for establishment of hete- rochromatin throughout a $20-\mathrm{kb}$ chromosomal domain containing mat2 and mat3 loci (Grewal and Klar 1997; Nakayama et al. 2000; Hall et al. 2002), which serve as donors of genetic information for the active mat 1 locus during mating-type switching (Klar 1989). Detailed analyses have uncovered the existence of at least two redundant mechanisms for the initial targeting of heterochromatin to the mating-type locus. In addition to the RNAi machinery acting through the $\mathrm{cenH}$ element (Hall et al. 2002), DNA-binding factors Atf1/Pcr1 belonging to the ATF/CREB protein family mediate heterochromatin nucleation at a distinct site (Jia et al. 2004a; Kim et al. 2004). Once nucleated, heterochromatin can spread across the entire silent mating-type interval, a process that depends on Swi6, Clr4, and histone deacetylases (HDACs) (Hall et al. 2002; Noma et al. 2004; Yamada et al. 2005). Heterochromatin assembled at the mating-type locus performs multiple functions. In addition to the wellrecognized function of heterochromatin in transcriptional silencing of developmentally important mating-type genes, it is also essential for recombinational suppression, which is believed to be critical for protecting the genomic 
integrity of the locus (Egel 1984; Klar and Bonaduce 1991; Thon et al. 1994; Grewal and Klar 1997).

Heterochromatin also mediates spreading of a protein complex, involved in mating-type switching, from an enhancer element located adjacent to the mat 3 locus to across the entire heterochromatic domain (Jia et al. 2004b). Importantly, this heterochromatin-mediated spreading occurs in a cell-type-specific manner only in $M$ cells but not in $P$ cells, which forms the basis for differential utilization of mat 2 or mat 3 loci during mating-type (mat l) switching (Jia et al. 2004b).

Sequences that share homology with centromeric repeats are also found embedded within subtelomeric regions. A partial or full-length copy of a RecQ-like gene (SPAC212.11) that has a cenH-like element in its coding region is believed to be essential for RNAi-mediated heterochromatin formation across subtelomeric regions (Cam et al. 2005; Kanoh et al. 2005; Hansen et al. 2006). Redundant mechanisms of heterochromatin nucleation are also operating at telomeres. In addition to RNAi machinery, a telomere repeat-binding protein Taz1, belonging to the TRF family (Cooper et al. 1997; Nimmo et al. 1998), is able to independently nucleate heterochromatin (Kanoh et al. 2005; Hansen et al. 2006). Although deletions of RNAi factors alone have no detectable effect on heterochromatin-mediated silencing at telomeres, due to redundancy in heterochromatin assembly mechanisms, mutations in RNAi factors result in defects in clustering of telomeres (Hall et al. 2003). This observation has led to suggestions that siRNAs promote higher-order chromatin organization by acting as a "glue" to hold dispersed repeats into a common structure (Hall et al. 2003).

Heterochromatin complexes are also found distributed at tandem $r D N A$ repeats, which are also targets of RNAi machinery (Cam et al. 2005). The mechanism of heterochromatin assembly and silencing at $r D N A$ in fission yeast remains to be fully explored. Recent studies suggest that RNAi and heterochromatin machineries are required for gene silencing and maintenance of genomic integrity at these loci (Thon and Verhein-Hansen 2000; Shankaranarayana et al. 2003; Bjerling et al. 2004; Cam et al. 2005).

In addition to repeat elements present at major heterochromatic regions, the fission yeast genome also contains several other repeat sequences, such as TF2 retrotransposons, WTF repeats, and solo long terminal repeats (LTRs) (Bowen et al. 2003). These repeat elements are not coated with heterochromatin components $\mathrm{H} 3 \mathrm{~K} 9 \mathrm{me}$ and Swi6 (Cam et al. 2005). Therefore, it seems that RNAi and heterochromatin machineries selectively target a specialized class of repeat elements, in particular $d g$ and $d h$ repeat elements found at all major heterochromatic loci.

\section{HETEROCHROMATIN DOMAIN BOUNDARIES}

A remarkable feature of heterochromatin protein complexes is their ability to spread in cis, resulting in epigenetic silencing of adjacent loci (Grewal and Moazed 2003; Talbert and Henikoff 2006). However, in their natural chromosomal contexts, heterochromatin domains are often surrounded by boundary DNA elements that prevent inappropriate spreading of repressive chromatin into euchromatic regions (Sun and Elgin 1999; West et al. 2002). Analysis of heterochromatin distribution in the fission yeast genome has revealed the existence of boundary elements surrounding the heterochromatin domains at all three centromeres and the silent mating-type locus (Partridge et al. 2000; Noma et al. 2001; Thon et al. 2002; Cam et al. 2005; Scott et al. 2006). Identical inverted repeat $(I R)$ elements surrounding the silent mating-type locus serve as heterochromatin domain boundaries (Fig. 1) (Noma et al. 2001; Thon et al. 2002). A marked decrease in H3K9me and Swi6 localization is observed coincident with the presence of $I R$ elements (Noma et al. 2001). Moreover, deletions of these elements result in spreading of heterochromatin to surrounding euchromatin domains (Noma et al. 2001). A sharp decrease in H3K9me and Swi6 levels is also observed at the pericentromeric heterochromatin domain boundaries, in which case clusters of $t R N A$ genes and/or inverted repeat elements, referred to as $I R C \mathrm{~s}$, have been suggested to serve as boundary elements (Fig. 1) (Partridge et al. 2000; Cam et al. 2005; Noma et al. 2006; Scott et al. 2006).

Our recent analyses suggest that $I R \mathrm{~s}$ and $t R N A$ genes might share a common boundary mechanism. We found that the core boundary sequences within $I R$ elements contain B-boxes (Noma et al. 2006), the high-affinity binding sites for the RNA polymerase III (pol III) transcription initiation factor TFIIIC, which is known to be important for transcription of $t R N A \mathrm{~s}$ (Huang et al. 2000; Huang and Maraia 2001). The binding of TFIIIC to IRs is essential for boundary function (Noma et al. 2006). In addition, TFIIIC binding to $t R N A$ gene clusters surrounding pericentromeric regions coincides precisely with a sharp decrease in H3K9me and Swi6 (Cam et al. 2005; Noma et al. 2006). A distinctive feature of $I R$ elements, however, is that TFIIIC binds to these loci without recruiting pol III. High levels of TFIIIC without pol III are also present at the so-called chromosome organizing clamps $(\mathrm{COCs})$ that are tethered to the nuclear peripheral compartment, at which TFIIIC is concentrated into $5-10$ bodies (Noma et al. 2006). According to a model, TFIIIC-bound sequences tether these loci to the nuclear periphery, thus creating a barrier against the spread of heterochromatin, in addition to facilitating higher-order genome organization.

Interestingly, TFIIIC does not bind to the IRC elements that surround cen 1 and cen3. Instead, these elements show preferential enrichment for a JmjC domain-containing antisilencing factor Epe1 (Zofall and Grewal 2006; see below), which we find is essential for transcription and barrier function of the IRC boundaries (Zofall and Grewal 2006).

\section{HISTONE-MODIFYING ENZYMES AND HETEROCHROMATIC TRANSCRIPTIONAL SILENCING}

Factors involved in modifications of histones have been shown to play an important role in the assembly of heterochromatic structures. In addition to the $\mathrm{Clr} 4$, which exists in a cullin 4 (Cul4)-Rik1-based E3 ubiquitin ligase complex (Hong et al. 2005; Horn et al. 2005; Jia et al. 2005; Thon et al. 2005), multiple HDACs (Sir2, Clr3, and Clr6) 
have been implicated in heterochromatin formation in fission yeast (Grewal et al. 1998; Freeman-Cook et al. 1999; Shankaranarayana et al. 2003; Ekwall 2005). Among these, Sir2 is an NAD-dependent histone deacetylase (FreemanCook et al. 1999; Shankaranarayana et al. 2003), whereas Clr3 and Clr6 belong to class 1 and class 2 family histone deacetylases, respectively (Grewal et al. 1998; Bjerling et al. 2002). Current evidence suggests multiple roles for HDACs in the establishment and maintenance of heterochromatic structures. Apart from setting up a histone modification pattern ("histone code") essential for localization of heterochromatin proteins, such as Swi6, HDAC proteins have been shown to be important for nucleation and spreading of heterochromatin complexes. This is best exemplified by studies at the mating-type region, in which case Clr3 HDAC cooperates with DNA-binding factors Atf1/Pcr1 to nucleate heterochromatin by mediating the targeting of Clr4 in addition to facilitating the assembly of "closed" chromatin across an entire heterochromatin domain (Jia et al. 2004a; Yamada et al. 2005).

Heterochromatin is believed to be highly condensed and inaccessible to factors involved in different aspects of DNA metabolism, including transcriptional machinery (Grewal and Elgin 2002). However, the mechanism of transcriptional silencing by heterochromatin is not fully understood. The oligomerization of Swi6/HP1 molecules bound to $\mathrm{H} 3 \mathrm{~K} 9 \mathrm{me}$ via their chromoshadow domain (Brasher et al. 2000; Cowieson et al. 2000) might facilitate chromatin condensation, resulting in silencing of underlying sequences. Nonetheless, recent studies have shown that Swi6/HP1 associates with target loci in a highly dynamic fashion (Cheutin et al. 2003, 2004; Festenstein et al. 2003). Our recent work suggests that Swi6 and another chromodomain protein, Chp2, bound to $\mathrm{H} 3 \mathrm{~K} 9 \mathrm{me}$ provide a loading platform for localization of HDAC Clr3, which in turn facilitates establishment of "closed" chromatin structure refractory to transcriptional machinery including RNA polymerase II (Yamada et al. 2005). In other words, chromodomain proteins bound to the H3K9me "anchor" serve as adapters for recruitment and/or spreading of regulatory proteins such as HDAC $\mathrm{Clr} 3$, to silence heterochromatic sequences, presumably through establishment of higher-order chromatin structures (Yamada et al. 2005).

\section{RNAI-MEDIATED HETEROCHROMATIC SILENCING}

RNAi was first described as a posttranscriptional silencing mechanism in which double-stranded RNA (dsRNA) triggers destruction of cognate RNAs (Fire et al. 1998). In this mechanism, long dsRNA is processed into siRNAs ranging in size from about 21 to 24 nucleotides by an RNase III enzyme Dicer (Hannon 2002). siRNAs are incorporated into an RNA-induced silencing complex (RISC) that, among other factors, contains Argonaute, which serves as a catalytic engine to mediate siRNAguided degradation of target mRNA (Liu et al. 2004; Meister et al. 2004; Rand et al. 2004). In some organisms, RNA-dependent RNA polymerases (RdRPs) have been shown to be important for RNAi silencing (Meister and
Tuschl 2004). As mentioned above, studies in fission yeast have implicated RNAi machinery, including Dicer (Dcr1), Argonaute (Ago1), and RdRP (Rdp1) in heterochromatic silencing (Hall et al. 2002; Reinhart and Bartel 2002; Volpe et al. 2002). Further genetic and biochemical studies have provided significant insights into the mechanism of RNAi-mediated heterochromatin assembly. An RNAi-induced initiation of transcriptional silencing (RITS) complex has been identified (Verdel et al. 2004). RITS contains Ago1, Chp1, and a protein named Tas 3 . In addition, RITS also contains siRNAs derived from $d g / d h$ repeats present at different heterochromatic loci (Verdel et al. 2004; Cam et al. 2005). Genome-mapping analyses have shown that components of RITS and Rdp1 are distributed throughout heterochromatic domains in a pattern almost identical to $\mathrm{H} 3 \mathrm{~K} 9 \mathrm{me}$ and Swi6 distribution (Cam et al. 2005). Moreover, stable binding of RITS to chromatin depends at least in part on the binding of Chp1 chromodomain to H3K9me (Noma et al. 2004). Deletion of an H3K9-specific methyltransferase Clr4 or Chp1 chromodomain results in delocalization of RITS from heterochromatic loci and concurrent defects in processing of repeat transcripts into siRNAs (Noma et al. 2004; Cam et al. 2005). RITS is also essential for the recruitment of the RDRC complex, which contains Rdp1, the RdRP activity of which is essential for siRNA production and heterochromatin assembly (Motamedi et al. 2004; Sugiyama et al. 2005).

We have proposed that RNAi-mediated heterochromatin assembly occurs via a self-reinforcing loop mechanism (Fig. 2) (Noma et al. 2004; Sugiyama et al. 2005). According to this model, siRNAs and/or DNA-binding factors mediate initial targeting of heterochromatin factors such as $\mathrm{Clr} 4$ to methylate $\mathrm{H} 3 \mathrm{~K} 9$. H3K9me allows stable binding of RITS across heterochromatin domains, which in turn mediates recruitment of RDRC to facilitate processing of repeat transcripts into siRNAs by Dcr1. siRNAs produced in cis can feed back to target additional heterochromatin complexes. The exact mechanism by which siRNAs target chromatin modifications is not clear. It has been suggested that RITS tethered to nascent transcripts via siRNAs might mediate recruitment of chromatin-modifying activities such as Clr4 or that siRNAs directly facilitate recruitment of the Clr4 complex to heterochromatic repeats (Verdel et al 2004; Jia et al. 2005).

\section{ARGONUATE SLICER FUNCTION IS ESSENTIAL FOR SIRNA PRODUCTION AND HETEROCHROMATIC SILENCING}

Argonaute proteins are defined by the presence of two conserved domains: PAZ (piwi-argonaute-zwille) and PIWI (P-element induced wimpy testis). Although the PAZ domain can be found in both Dicer and Argonaute proteins, the PIWI domain is present only in Argonaute proteins (Meister and Tuschl 2004). Recent studies have shown that the PIWI domain is structurally related to RNase H ribonucleases (Song et al. 2004; Yuan et al. 2005), and biochemical mutagenesis analyses of mammalian Argonaute 2 protein showed that the cryptic 


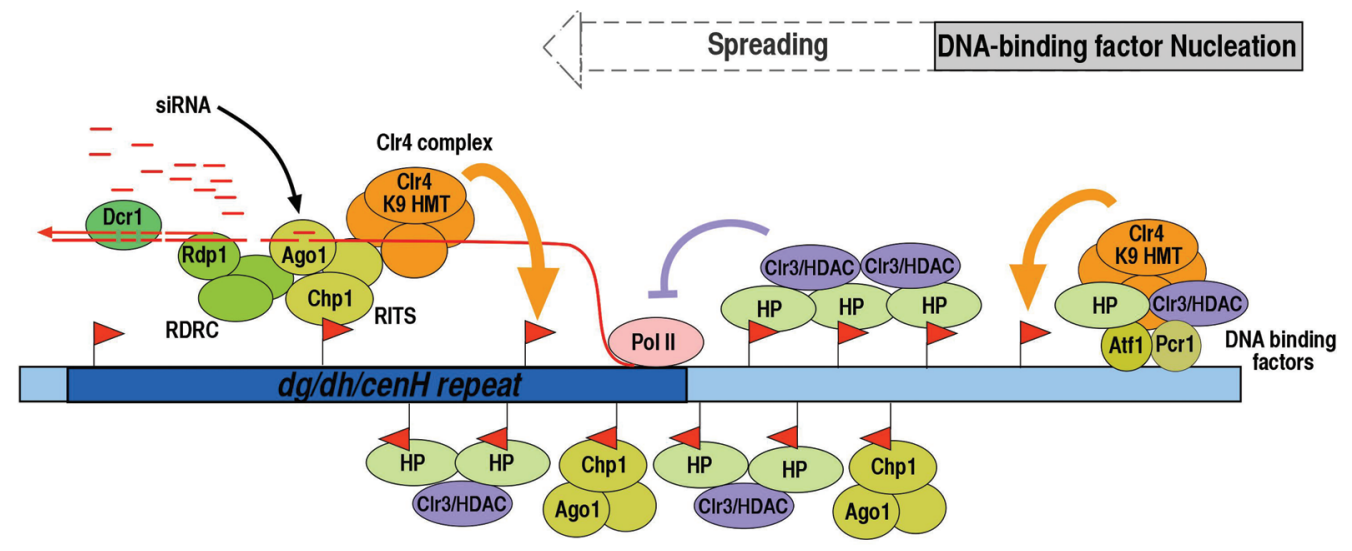

RNAi Nucleation Spreading

Figure 2. Redundant RNAi- and DNA-based mechanisms contribute to heterochromatin assembly. Sequence-specific DNA-binding proteins such as Atf1/Pcr1 cooperate with heterochromatin assembly factors including HDAC Clr3 to recruit Clr4 histone methyltransferase (HMT) to a specific site, and RNAi machinery targets heterochromatin factors to the $d g / d h / c e n H$ repeat elements. RNAimediated heterochromatin assembly involves processing of repeat transcripts by RITS, RDRC, and Dicer into siRNAs that are believed to mediate targeting of Clr4. Methylation of H3K9 by Clr4 (indicated by red flags) is not only necessary for RITS to stay stably associated with heterochromatic loci via Chp1 chromodomain, but it also recruits Swi6 and Chp2 (HPs), which, among other factors, mediate spreading of $\mathrm{Clr} 3 \mathrm{HDAC}$ implicated in limiting pol II accessibility to heterochromatic sequences. Spreading of H3K9me-Swi6 also allows RNAi machinery to spread beyond the initial nucleation site, potentially providing an opportunity to exert control over sequences incapable of nucleating heterochromatin.

RNase $\mathrm{H}$ domain is essential for Slicer activity of the RISC complex (Liu et al. 2004; Meister et al. 2004; Rand et al. 2004).

We sought to explore whether the endonucleolytic activity associated with the PIWI domain of Argonaute proteins is required for heterochromatic silencing. The aspartate residues 580 and 651 of a single fission yeast Ago1 protein were identified by a structure-alignment approach as a part of the highly conserved catalytic carboxylate triad "DDE," known to be conserved in all RNase H fold nucleases (Fig. 3a) (Yang and Steitz 1995; Song et al. 2004; Yuan et al. 2005). We constructed fission yeast strains carrying mutations in either of two conserved aspartate residues (i.e., D580A or D651A). Immunoblotting assay showed that mutant protein levels are comparable to that of wild-type Agol protein (Fig. $3 \mathrm{c})$. However, we found that mutations abolished silencing of a $\mathrm{ura}^{+}$reporter gene (otr $1:: \mathrm{ura}^{+}{ }^{+}$) inserted at the pericentromeric repeats region of cenl (Fig. 3b). To test whether the loss of silencing in mutant cells is caused by defects in heterochromatin assembly at centromeres, we performed chromatin immunoprecipitation (ChIP) assays to examine the status of $\mathrm{H} 3 \mathrm{~K} 9 \mathrm{me}$ and Swi6 localization at otr $1::$ ura $^{+}$. These analyses revealed that localization of both $\mathrm{H} 3 \mathrm{~K} 9 \mathrm{me}$ and Swi6 at centromeric otr $1::$ ura $^{+}$was severely affected in both ago ${ }^{D 580 A}$ and $\operatorname{agol}^{D 651 \mathrm{~A}}$ mutants (Fig. 4c). On the basis of these analyses, we conclude that the catalytic activity of Agol protein might be essential for the heterochromatin assembly at centromeres.

We next investigated whether ago $1^{D 580 A}$ and ago $1^{D 651 A}$ mutations also affect silencing and heterochromatin assembly at the naturally silenced $d g / d h$ centromeric repeat elements. Reverse transcriptase-polymerase chain reaction (RT-PCR) analyses of total RNA showed that transcripts originating from the $d g$ centromeric repeat elements could be readily detected in the agol mutant strains (Fig. 4b). We also observed increased accumulation of transcripts derived from cenH in ggol $^{D 580 A}$ and ago $^{D 651 A}$ mutants even though comparable levels of wild-type and mutant Agol proteins could be ChIPed at this site (our unpublished data). These results suggest that the processing of repeat transcripts into heterochromatic siRNAs requires the conserved catalytic aspartate residues of Ago1 PIWI domain. Indeed, although siRNAs could be readily detected in wild-type background cells, there were no detectable siRNAs present in mutant background cells (Fig. 4a). Intriguingly, we found that mutations in Ago1 had only a minor effect on $\mathrm{H} 3 \mathrm{~K} 9$ me and Swi6 localization at the repeat elements (Fig. 4d), despite severely reduced levels of $\mathrm{H} 3 \mathrm{~K} 9 \mathrm{me}$ and Swi6 observed at the otr $1::$ ura $^{+}$ reporter construct (Fig. 4c). Significant levels of H3K9me and Swi6 remaining at the repeat elements in agol mutants are consistent with our previous results showing that an RNAi-independent pathway involving Clr3 HDAC contributes to heterochromatin nucleation by mediating targeting of Clr4 to centromeric repeats (Yamada et al. 2005). Based on these results and previous studies (Sadaie et al. 2004; Yamada et al. 2005), cells defective in siRNA production can target $\mathrm{H} 3 \mathrm{~K} 9$ me to centromeric repeat elements, but siRNA production is necessary for spreading of heterochromatin complexes into the otr $1::$ ura $4^{+}$gene. How might siRNAs facilitate local spreading of heterochromatin? In light of evidence showing that assembly of heterochromatic structures requires RNAs (Maison et al. 2002; Muchardt et al. 2002; Hall et al. 2003), one pos- 
a
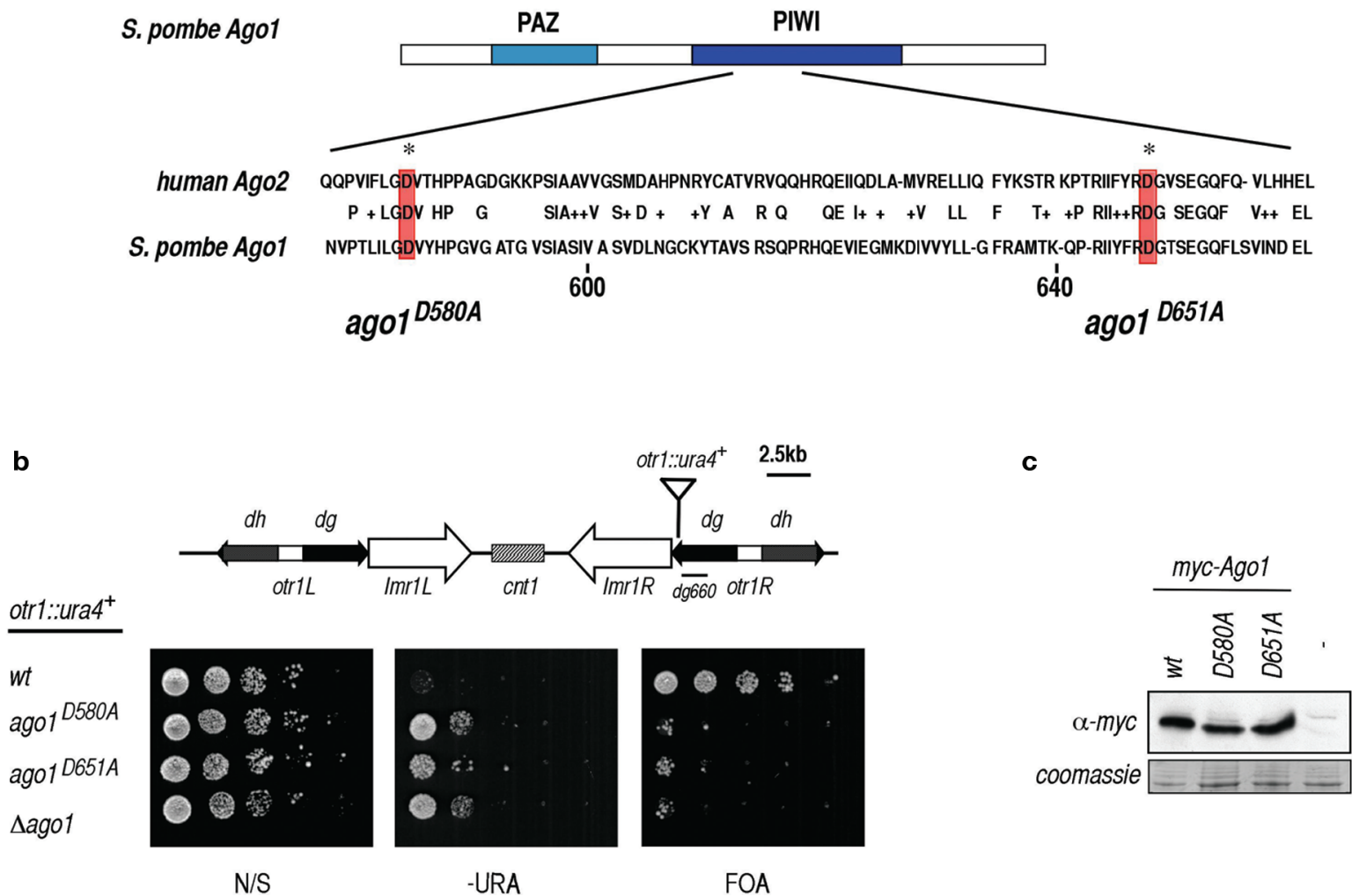

Figure 3. Mutations in conserved Ago1 residues implicated in "slicer" activity of Argonaute family proteins affect heterochromatin silencing. (a) Alignment of PIWI domains of human Ago2 and S. pombe Ago1 proteins is shown. The aspartate residues highlighted in red are known to be essential for slicing activity of human Ago2 protein. (b) Mutations of conserved aspartate residues (D580A and D651A) abolish heterochromatic silencing at centromeres. Effects of mutations on silencing of a $\mathrm{ura}^{+}$reporter gene inserted at outer pericentromeric repeats (otr $1: \mathrm{ura}^{+}$) were investigated by performing a serial dilution plating assay. Tenfold serial dilutions of indicated cultures were plated on nonselective (N/S), uracil-deficient (-ura), and counterselective (5-FOA) medium and allowed to grow at $32^{\circ} \mathrm{C}$ for 2 days. (c) Expression of wild-type and mutant Agol proteins was compared by western blot analysis. Whole-cell extracts prepared from cells expressing wild-type or mutant proteins tagged at the amino terminus by a myc epitope tag were examined by western blotting with anti-myc(A14) antibody (Santa Cruz Biotechnology).

sibility is that siRNAs produced in cis by the RNAi machinery have a structural role in organizing repeat elements into higher-order condensed structures that, in addition to mediating silencing of the repeat elements, facilitate local spreading of heterochromatin complexes.

\section{SWI6/HP1 MEDIATES RECRUITMENT OF BOTH SILENCING AND ANTISILENCING FACTORS}

RNA pol II transcribes centromeric $d g / d h$ repeats even in the presence of heterochromatin (Cam et al. 2005; Djupedal et al. 2005). Since transcription of repeats is essential for generation of siRNA precursors, mutations in pol II impair RNAi-mediated heterochromatin assembly (Djupedal et al. 2005; Kato et al. 2005). However, the mechanism by which pol II gains access to sequences heavily coated with heterochromatin was not clear. Our recent work revealed that a JmjC domain-containing protein Epe1 facilitates pol II accessibility and transcription of heterochromatic repeats (Zofall and Grewal 2006). Epe1 is required for transcription of repeats specifically in a heterochromatin context. However, in mutant cells defective in heterochromatin silencing, Epe1 is dispens- able for transcription of repeats. Thus, it seems that Epe1 is specifically required for pol II transcription in the presence of repressive heterochromatin.

Epe1 was first identified in a screen for factors that negatively regulate the integrity of heterochromatin (Ayoub et al. 2003). Whereas loss of Epe1 enhances heterochromatic silencing and results in efficient spreading of heterochromatin into euchromatic regions, its overexpression destabilizes heterochromatin that correlates with increased histone acetylation and H3K4me (Ayoub et al. 2003). We found that Epe1 is paradoxically enriched throughout heterochromatic domains (Zofall and Grewal 2006). More surprisingly, the recruitment of Epe1 to heterochromatic loci is dependent on its interaction with Swi6. Epe1 binds directly to Swi6 in vitro, and these proteins form a complex in vivo. These observations, together with our recent work showing that Swi6 recruits Clr3 HDAC to restrict pol II accessibility to heterochromatic repeats (Yamada et al. 2005), suggest that Swi6 serves as an oscillator of heterochromatic transcription by directing recruitment of both silencing and antisilencing factors, the balance of which might be critical for proper maintenance of heterochromatic structures (Fig. 5). 
a

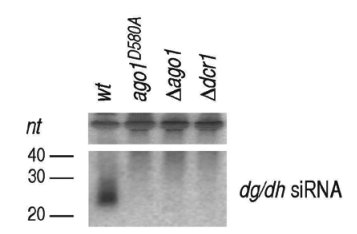

c

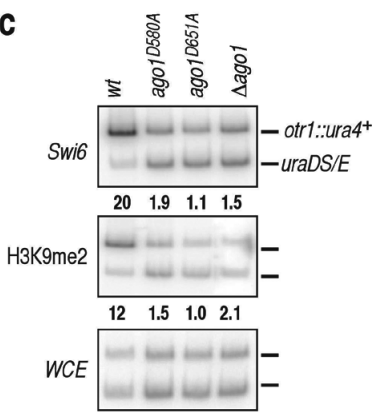

b
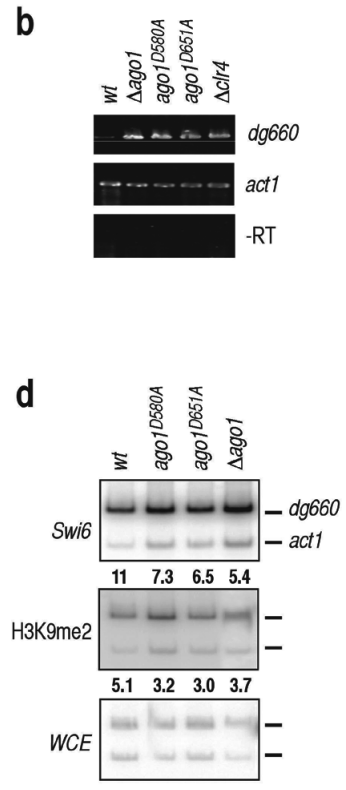

Figure 4. Effects of mutations in conserved PIWI domain of Agol on siRNA production, repeat silencing, and heterochromatin formation. (a) Agol mutants are defective in siRNA production. $20 \mu \mathrm{g}$ of small RNA fraction was resolved on $15 \%$ urea-PAGE gel and transferred to Hybond ${ }^{\mathrm{N}+}$ membrane. Membrane was hybridized with single-stranded RNA probes transcribed with $\alpha-{ }^{32} \mathrm{P}$-labeled UTP and hydrolyzed to lengths averaging 50 nucleotides (Hamilton and Baulcombe 1999). (b) Mutations in the PIWI domain of Agol result in accumulation of transcripts derived from centromeric repeats. The levels of $d g$ repeat transcripts were analyzed by RT-PCR using total RNA prepared from indicated strains. The same RNA samples were also used to amplify control act 1 transcript in the presence and absence of reverse transcriptase. PCR products were separated on agarose gel and were visualized by ethidium bromide staining. $(c, d)$ Loss of silencing in Ago1 mutants correlates with defects in heterochromatin assembly. Chromatin immunoprecipitation (ChIP) analysis was used to measure levels of Swi6 or $\mathrm{H} 3 \mathrm{~K} 9 \mathrm{me}$ at $d g$ centromeric repeats $(d)$ or $\operatorname{otr} 1: \because \mathrm{urat}^{+}(\mathrm{c})$. ChIP was performed and analyzed as described previously (Nakayama et al. 2000) using antibodies against Swi6 and dimethylated H3K9 (Upstate Biotechnology).

The mechanism by which Epe1 counteracts heterochromatic silencing is not known. Since several JmjC domain proteins have been shown to catalyze histone demethylation (Tsukada et al. 2005; Fodor et al. 2006; Klose et al. 2006; Whetstine et al. 2006), it is formally possible that Epe1 affects heterochromatin stability through removal of the repressive lysine methylation mark. However, no such activity has been detected for Epe1 (Tsukada et al. 2005). A possibility remains that Epe1 modulates chromatin via a yet undefined mechanism.

\section{CONCLUDING REMARKS}

Studies from several systems suggest an important role for RNAi machinery in assembly of repressive chromatin structures (Mochizuki and Gorovsky 2004; Chan et al. 2005; Matzke and Birchler 2005). Recent investigation into the role of RNAi in heterochromatin formation in fission

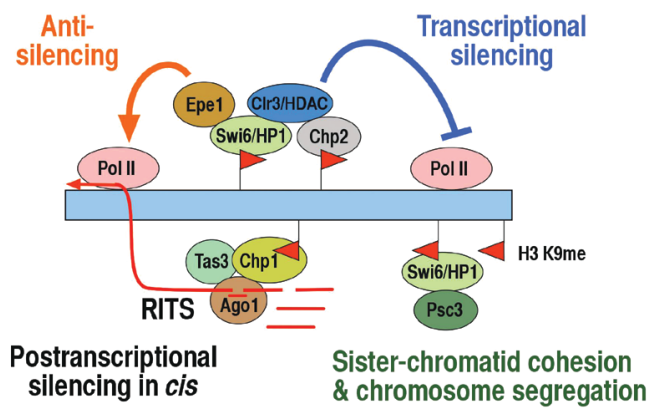

Figure 5. Binding of chromodomain proteins to $\mathrm{H} 3 \mathrm{~K} 9$ me establishes a platform for recruitment of distinct activities. H3K9me nucleated by DNA-binding factors or RNAi pathway tethers chromodomain proteins Chp1, Chp2, and Swi6/HP1, which mediate targeting of distinct activities involved in heterochromatin maintenance. In addition to recruiting cohesin, which promotes proper chromosome segregation and chromatid cohesion, Swi6/HP1 targets opposing activities of HDAC Clr3 and jmjC protein Epe1. Whereas HDAC Clr3 limits pol II accessibility and transcription, Epe1 facilitates transcription and promotes pol II association with heterochromatic regions. Chromodomain protein Chp1 is a component of RITS that allows RNAi machinery to act in cis to process repeat transcripts into siRNAs.

yeast has shown that RNAi factors localize throughout heterochromatin domains and that their localization across these domains is mediated by components of heterochromatin such as H3K9me and Swi6/HP1 (Noma et al. 2004; Cam et al. 2005; Sugiyama et al. 2005). These studies suggest that RNAi-mediated heterochromatin assembly occurs via a self-reinforcing loop mechanism in which chromatinassociated RITS complex serves as a core for stable binding of RNAi factors, including RdRP Rdp1, which cooperates to process repeat transcripts into siRNAs, essential for further strengthening of heterochromatic structures (Noma et al. 2004; Sugiyama et al. 2005). Rdp1 is a functional RNA-dependent RNA polymerase, and its RdRP activity is essential for generation of siRNAs (Motamedi et al. 2004; Sugiyama et al. 2005). Similarly, Dicer is required for the processing of repeat transcripts into siRNAs. However, the precise function of the Agol protein remained unexplored. Our analysis suggests that mutations in conserved Agol residues that are known to be essential for Slicer activity of Argonaute family proteins (Liu et al. 2004; Meister et al. 2004; Rand et al. 2004) severely affect processing of repeat transcripts into siRNAs, resulting in defects in heterochromatin assembly at centromeres. These results indicate that siRNA-guided cleavage of nascent repeat transcripts by Agol is likely an essential part of the siRNA production cycle. The cleaved transcripts might be a preferential substrate for Rdp1-mediated conversion into dsRNA or, alternatively, cleavage interferes with maturation of transcript such as polyadenylation and capping, making them preferential targets for Rdp1. Future work will address these possibilities.

An important characteristic of heterochromatic structures is their ability to exert a repressive influence on transcription of underlying sequences. It is therefore surprising that continuous transcription of heterochromatic repeats by pol II is required for RNAi-mediated het- 
erochromatin assembly. Our work suggests that conserved heterochromatin protein Swi6/HP1 not only provides a platform for recruitment/spreading of silencing factors, but also targets antisilencing factors capable of facilitating pol II accessibility to sequences coated with heterochromatin. Therefore, proper maintenance of heterochromatin seems to require dynamic equilibrium of opposing chromatin-modifying activities such as HDACs that promote assembly of condensed higher-order structures and JmjC domain protein Epel capable of destabilizing these structures. Future investigations into mechanisms governing the relative influence of these activities is expected to provide insights into heterochromatin-mediated epigenetic reprogramming of target loci.

\section{ACKNOWLEDGMENTS}

We thank members of the Grewal laboratory for helpful discussions, and Hugh Cam and Ken-ichi Noma for help in manuscript preparation. Research in our laboratory is supported by the Intramural Research Program of the National Institutes of Health, National Cancer Institute.

\section{REFERENCES}

Ayoub N., Noma K., Isaac S., Kahan T., Grewal S.I., and Cohen A. 2003. A novel jmjC domain protein modulates heterochromatization in fission yeast. Mol. Cell. Biol. 23: 4356.

Bannister A.J., Zegerman P., Partridge J.F., Miska E.A., Thomas J.O., Allshire R.C., and Kouzarides T. 2001. Selective recognition of methylated lysine 9 on histone H3 by the HP1 chromo domain. Nature 410: 120.

Bernard P., Maure J.F., Partridge J.F., Genier S., Javerzat J.P., and Allshire R.C. 2001. Requirement of heterochromatin for cohesion at centromeres. Science 294: 2539.

Birchler J.A., Bhadra M.P., and Bhadra U. 2000. Making noise about silence: Repression of repeated genes in animals. Curr. Opin. Genet. Dev. 10: 211.

Bjerling P. and Ekwall K. 2002. Centromere domain organization and histone modifications. Braz. J. Med. Biol. Res. 35: 499.

Bjerling P., Ekwall K., Egel R., and Thon G. 2004. A novel type of silencing factor, Clr2, is necessary for transcriptional silencing at various chromosomal locations in the fission yeast Schizosaccharomyces pombe. Nucleic Acids Res. 32: 4421.

Bjerling P., Silverstein R.A., Thon G., Caudy A., Grewal S., and Ekwall K. 2002. Functional divergence between histone deacetylases in fission yeast by distinct cellular localization and in vivo specificity. Mol. Cell. Biol. 22: 2170.

Bowen N.J., Jordan I.K., Epstein J.A., Wood V., and Levin H.L. 2003. Retrotransposons and their recognition of pol II promoters: A comprehensive survey of the transposable elements from the complete genome sequence of Schizosaccharomyces pombe. Genome Res. 13: 1984.

Brasher S.V., Smith B.O., Fogh R.H., Nietlispach D., Thiru A., Nielsen P.R., Broadhurst R.W., Ball L.J., Murzina N.V., and Laue E.D. 2000. The structure of mouse HP1 suggests a unique mode of single peptide recognition by the shadow chromo domain dimer. EMBO J. 19: 1587.

Cam H. and Grewal S.I. 2004. RNA interference and epigenetic control of heterochromatin assembly in fission yeast. Cold Spring Harbor Symp. Quant. Biol. 69: 419.

Cam H., Sugiyama T., Chen E.S., Chen X., Fitzgerald P., and Grewal S.I. 2005. Comprehensive analysis of heterochromatin- and RNAi-mediated epigenetic control of the fission yeast genome. Nat. Genet. 37: 809.

Chan S.W., Henderson I.R., and Jacobsen S.E. 2005. Gardening the genome: DNA methylation in Arabidopsis thaliana. Nat. Rev. Genet. 6: 351 .

Cheutin T., Gorski S.A., May K.M., Singh P.B., and Misteli T. 2004. In vivo dynamics of Swi6 in yeast: Evidence for a stochastic model of heterochromatin. Mol. Cell. Biol. 24: 3157.

Cheutin T., McNairn A.J., Jenuwein T., Gilbert D.M., Singh P.B., and Misteli T. 2003. Maintenance of stable heterochromatin domains by dynamic HP1 binding. Science 299: 721.

Chikashige Y., Kinoshita N., Nakaseko Y., Matsumoto T., Murakami S., Niwa O., and Yanagida M. 1989. Composite motifs and repeat symmetry in $S$. pombe centromeres: Direct analysis by integration of NotI restriction sites. Cell 57: 739 .

Clarke L., Amstutz H., Fishel B., and Carbon J. 1986. Analysis of centromeric DNA in the fission yeast Schizosaccharomyces pombe. Proc. Natl. Acad. Sci. 83: 8253.

Cooper J.P., Nimmo E.R., Allshire R.C., and Cech T.R. 1997. Regulation of telomere length and function by a Myb-domain protein in fission yeast. Nature 385: 744 .

Cowieson N.P., Partridge J.F., Allshire R.C., and McLaughlin P.J. 2000. Dimerisation of a chromo shadow domain and distinctions from the chromodomain as revealed by structural analysis. Curr. Biol. 10: 517.

Djupedal I., Portoso M., Spahr H., Bonilla C., Gustafsson C.M., Allshire R.C., and Ekwall K. 2005. RNA Pol II subunit Rpb7 promotes centromeric transcription and RNAi-directed chromatin silencing. Genes Dev. 19: 2301.

Ebert A., Lein S., Schotta G., and Reuter G. 2006. Histone modification and the control of heterochromatic gene silencing in Drosophila. Chromosome Res. 14: 377.

Egel R. 1984. Two tightly linked silent cassettes in the matingtype region of Schizosaccharomyces pombe. Curr. Genet. 8: 199.

Ekwall K. 2005. Genome-wide analysis of HDAC function. Trends Genet. 21: 608.

Festenstein R., Pagakis S.N., Hiragami K., Lyon D., Verreault A., Sekkali B., and Kioussis D. 2003. Modulation of heterochromatin protein 1 dynamics in primary mammalian cells. Science 299: 719.

Fire A., Xu S., Montgomery M.K., Kostas S.A., Driver S.E., and Mello C.C. 1998. Potent and specific genetic interference by double-stranded RNA in Caenorhabditis elegans. Nature 391: 806 .

Fodor B.D., Kubicek S., Yonezawa M., O'Sullivan R.J., Sengupta R., Perez-Burgos L., Opravil S., Mechtler K., Schotta G., and Jenuwein T. 2006. Jmjd2b antagonizes H3K9 trimethylation at pericentric heterochromatin in mammalian cells. Genes Dev. 20: 1557.

Freeman-Cook L.L., Sherman J.M., Brachmann C.B., Allshire R.C., Boeke J.D., and Pillus L. 1999. The Schizosaccharomyces pombe hst4(+) gene is a SIR2 homologue with silencing and centromeric functions. Mol. Biol. Cell 10: 3171

Grewal S.I. and Elgin S.C. 2002. Heterochromatin: New possibilities for the inheritance of structure. Curr. Opin. Genet. Dev. 12: 178 .

Grewal S.I. and Klar A.J. 1997. A recombinationally repressed region between mat 2 and mat3 loci shares homology to centromeric repeats and regulates directionality of mating-type switching in fission yeast. Genetics 146: 1221 .

Grewal S.I. and Moazed D. 2003. Heterochromatin and epigenetic control of gene expression. Science 301: 798.

Grewal S.I., Bonaduce M.J., and Klar A.J. 1998. Histone deacetylase homologs regulate epigenetic inheritance of transcriptional silencing and chromosome segregation in fission yeast. Genetics 150: 563.

Hahnenberger K.M., Carbon J., and Clarke L. 1991. Identification of DNA regions required for mitotic and meiotic functions within the centromere of Schizosaccharomyces pombe chromosome I. Mol. Cell. Biol. 11: 2206.

Hall I.M. and Grewal S. I. 2003. Structure and function of heterochromatin: Implications for epigenetic gene silencing and genome organization. In RNAi: A guide to gene silencing (ed. G.J. Hannon), p. 205. Cold Spring Harbor Laboratory Press, Cold Spring Harbor, New York. 
Hall I.M., Noma K., and Grewal S.I. 2003. RNA interference machinery regulates chromosome dynamics during mitosis and meiosis in fission yeast. Proc. Natl. Acad. Sci. 100: 193.

Hall I.M., Shankaranarayana G.D., Noma K., Ayoub N., Cohen A., and Grewal S.I. 2002. Establishment and maintenance of a heterochromatin domain. Science 297: 2232.

Hamilton A.J. and Baulcombe D.C. 1999. A species of small antisense RNA in posttranscriptional gene silencing in plants. Science 286: 950.

Hannon G.J. 2002. RNA interference. Nature 418: 244.

Hansen K.R., Ibarra P.T., and Thon G. 2006. Evolutionary-conserved telomere-linked helicase genes of fission yeast are repressed by silencing factors, RNAi components and the telomere-binding protein Taz1. Nucleic Acids Res. 34: 78.

Henikoff S. 2000. Heterochromatin function in complex genomes. Biochim. Biophys. Acta 1470: 1.

Hiragami K. and Festenstein R. 2005. Heterochromatin protein 1: A pervasive controlling influence. Cell. Mol. Life Sci. 62: 2711.

Hong E.E., Villen J., Gerace E.L., Gygi S.P., and Moazed D. 2005. A Cullin E3 ubiquitin ligase complex associates with Rik1 and the Clr4 histone H3-K9 methyltransferase and is required for RNAi-mediated heterochromatin formation. $R N A$ Biol. 2: 106.

Horn P.J., Bastie J.N., and Peterson C.L. 2005. A Rik1-associated, cullin-dependent E3 ubiquitin ligase is essential for heterochromatin formation. Genes Dev. 19: 1705.

Huang Y. and Maraia R.J. 2001. Comparison of the RNA polymerase III transcription machinery in Schizosaccharomyces pombe, Saccharomyces cerevisiae and human. Nucleic Acids Res. 29: 2675.

Huang Y., Hamada M., and Maraia R.J. 2000. Isolation and cloning of four subunits of a fission yeast TFIIIC complex that includes an ortholog of the human regulatory protein TFIIICbeta. J. Biol. Chem. 275: 31480 .

Huisinga K.L., Brower-Toland B., and Elgin S.C. 2006. The contradictory definitions of heterochromatin: Transcription and silencing. Chromosoma 115: 110 .

Ivanova A.V., Bonaduce M.J., Ivanov S.V., and Klar A.J. 1998. The chromo and SET domains of the Clr4 protein are essential for silencing in fission yeast. Nat. Genet. 19: 192.

Jia S., Kobayashi R., and Grewal S.I. 2005. Ubiquitin ligase component $\mathrm{Cul} 4$ associates with Clr4 histone methyltransferase to assemble heterochromatin. Nat. Cell Biol. 7: 1007.

Jia S., Noma K., and Grewal S.I. 2004a. RNAi-independent heterochromatin nucleation by the stress-activated ATF/CREB family proteins. Science 304: 1971.

Jia S., Yamada T., and Grewal S.I. 2004b. Heterochromatin regulates cell type-specific long-range chromatin interactions essential for directed recombination. Cell 119: 469.

Kanoh J., Sadaie M., Urano T., and Ishikawa F. 2005. Telomere binding protein Tazl establishes Swi6 heterochromatin independently of RNAi at telomeres. Curr. Biol. 15: 1808.

Kato H., Goto D.B., Martienssen R.A., Urano T., Furukawa K., and Murakami Y. 2005. RNA polymerase II is required for RNAi-dependent heterochromatin assembly. Science 309: 467.

Kim H.S., Choi E.S., Shin J.A., Jang Y.K., and Park S.D. 2004. Regulation of Swi6/HP1-dependent heterochromatin assembly by cooperation of components of the mitogen-activated protein kinase pathway and a histone deacetylase Clr6. J. Biol. Chem. 279: 42850.

Klar A.J. 1989. The interconversion of yeast mating type: Saccharomyces cerevisiae and Schizosaccharomyces pombe. In Mobile DNA (ed. D.E. Berg and M.W. Howe), p. 671. American Society for Microbiology, Washington, D.C.

Klar A.J. and Bonaduce M.J. 1991. swi6, a gene required for mating-type switching, prohibits meiotic recombination in the mat2-mat3 "cold spot" of fission yeast. Genetics 129: 1033.

Klose R.J., Yamane K., Bae Y., Zhang D., Erdjument-Bromage H., Tempst P., Wong J., and Zhang Y. 2006. The transcriptional repressor JHDM3A demethylates trimethyl histone $\mathrm{H} 3$ lysine 9 and lysine 36. Nature 442: 312.
Liu J., Carmell M.A., Rivas F.V., Marsden C.G., Thomson J.M., Song J.J., Hammond S.M., Joshua-Tor L., and Hannon G.J. 2004. Argonaute2 is the catalytic engine of mammalian RNAi. Science 305: 1437.

Lorentz A., Heim L., and Schmidt H. 1992. The switching gene swi6 affects recombination and gene expression in the mating-type region of Schizosaccharomyces pombe. Mol. Gen. Genet. 233: 436

Maison C., Bailly D., Peters A.H., Quivy J.P., Roche D., Taddei A., Lachner M., Jenuwein T., and Almouzni G. 2002. Higher-order structure in pericentric heterochromatin involves a distinct pattern of histone modification and an RNA component. Nat. Genet. 30: 329.

Matzke M.A. and Birchler J.A. 2005. RNAi-mediated pathways in the nucleus. Nat. Rev. Genet. 6: 24.

Meister G. and Tuschl T. 2004. Mechanisms of gene silencing by double-stranded RNA. Nature 431: 343 .

Meister G., Landthaler M., Patkaniowska A., Dorsett Y., Teng G., and Tuschl T. 2004. Human Argonaute2 mediates RNA cleavage targeted by miRNAs and siRNAs. Mol. Cell 15: 185 .

Mochizuki K. and Gorovsky M.A. 2004. Small RNAs in genome rearrangement in Tetrahymena. Curr. Opin. Genet. Dev. 14: 181

Motamedi M.R., Verdel A., Colmenares S.U., Gerber S.A., Gygi S.P., and Moazed D. 2004. Two RNAi complexes, RITS and RDRC, physically interact and localize to noncoding centromeric RNAs. Cell 119: 789.

Muchardt C., Guilleme M., Seeler J.S., Trouche D., Dejean A., and Yaniv M. 2002. Coordinated methyl and RNA binding is required for heterochromatin localization of mammalian HP1alpha. EMBO Rep. 3: 975.

Nakayama J., Klar A.J., and Grewal S.I. 2000. A chromodomain protein, Swi6, performs imprinting functions in fission yeast during mitosis and meiosis. Cell 101: 307.

Nakayama J., Rice J.C., Strahl B.D., Allis C.D., and Grewal S.I. 2001. Role of histone H3 lysine 9 methylation in epigenetic control of heterochromatin assembly. Science 292: 110.

Nimmo E.R., Pidoux A.L., Perry P.E., and Allshire R.C. 1998. Defective meiosis in telomere-silencing mutants of Schizosaccharomyces pombe. Nature 392: 825.

Noma K., Allis C.D., and Grewal S.I. 2001. Transitions in distinct histone $\mathrm{H} 3$ methylation patterns at the heterochromatin domain boundaries. Science 293: 1150.

Noma K., Cam H.P., Maraia R.J., and Grewal S.I. 2006. A role for TFIIIC transcription factor complex in genome organization. Cell 125: 859.

Noma K., Sugiyama T., Cam H., Verdel A., Zofall M., Jia S., Moazed D., and Grewal S.I. 2004. RITS acts in cis to promote RNA interference-mediated transcriptional and posttranscriptional silencing. Nat. Genet. 36: 1174.

Nonaka N., Kitajima T., Yokobayashi S., Xiao G., Yamamoto M., Grewal S.I., and Watanabe Y. 2002. Recruitment of cohesin to heterochromatic regions by Swi6/HP1 in fission yeast. Nat. Cell Biol. 4: 89.

Partridge J.F., Borgstrom B., and Allshire R.C. 2000. Distinct protein interaction domains and protein spreading in a complex centromere. Genes Dev. 14: 783 .

Pidoux A.L. and Allshire R.C. 2004. Kinetochore and heterochromatin domains of the fission yeast centromere. Chromosome Res. 12: 521.

Rand T.A., Ginalski K., Grishin N.V., and Wang X. 2004. Biochemical identification of Argonaute 2 as the sole protein required for RNA-induced silencing complex activity. Proc. Natl. Acad. Sci. 101: 14385.

Rea S., Eisenhaber F., O'Carroll D., Strahl B.D., Sun Z.W., Schmid M., Opravil S., Mechtler K., Ponting C.P., Allis C.D., and Jenuwein T. 2000. Regulation of chromatin structure by site-specific histone $\mathrm{H} 3$ methyltransferases. Nature 406: 593.

Reinhart B.J. and Bartel D.P. 2002. Small RNAs correspond to centromere heterochromatic repeats. Science 297: 1831.

Sadaie M., Iida T., Urano T., and Nakayama J. 2004. A chromodomain protein, Chp1, is required for the establishment of heterochromatin in fission yeast. EMBO J. 23: 3825. 
Saitoh S., Takahashi K., and Yanagida M. 1997. Mis6, a fission yeast inner centromere protein, acts during G1/S and forms specialized chromatin required for equal segregation. Cell 90: 131.

Scott K.C., Merrett S.L., and Willard H.F. 2006. A heterochromatin barrier partitions the fission yeast centromere into discrete chromatin domains. Curr. Biol. 16: 119.

Shankaranarayana G.D., Motamedi M.R., Moazed D., and Grewal S.I. 2003. Sir2 regulates histone H3 lysine 9 methylation and heterochromatin assembly in fission yeast. Curr. Biol. 13: 1240.

Song J.J., Smith S.K., Hannon G.J., and Joshua-Tor L. 2004. Crystal structure of Argonaute and its implications for RISC slicer activity. Science 305: 1434.

Sugiyama T., Cam H., Verdel A., Moazed D., and Grewal S.I. 2005. RNA-dependent RNA polymerase is an essential component of a self-enforcing loop coupling heterochromatin assembly to siRNA production. Proc. Natl. Acad. Sci. 102: 152.

Sun F.L. and Elgin S.C. 1999. Putting boundaries on silence. Cell 99: 459.

Takahashi K., Chen E.S., and Yanagida M. 2000. Requirement of Mis6 centromere connector for localizing a CENP-A-like protein in fission yeast. Science 288: 2215.

Talbert P.B. and Henikoff S. 2006. Spreading of silent chromatin: Inaction at a distance. Nat. Rev. Genet. 7: 793.

Thon G. and Verhein-Hansen J. 2000. Four chromo-domain proteins of Schizosaccharomyces pombe differentially repress transcription at various chromosomal locations. Genetics 155: 551.

Thon G., Cohen A., and Klar A.J. 1994. Three additional linkage groups that repress transcription and meiotic recombination in the mating-type region of Schizosaccharomyces pombe. Genetics 138: 29.

Thon G., Bjerling P., Bunner C.M., and Verhein-Hansen J. 2002. Expression-state boundaries in the mating-type region of fission yeast. Genetics 161: 611 .
Thon G., Hansen K.R., Altes S.P., Sidhu D., Singh G., Verhein-Hansen J., Bonaduce M.J., and Klar A.J. 2005. The Clr7 and Clr8 directionality factors and the Pcu 4 cullin mediate heterochromatin formation in the fission yeast Schizosaccharomyces pombe. Genetics 171: 1583.

Tsukada Y.I., Fang J., Erdjument-Bromage H., Warren M.E., Borchers C.H., Tempst P., and Y. Zhang. 2005. Histone demethylation by a family of JmjC domain-containing proteins. Nature 439: 811.

Verdel A., Jia S., Gerber S., Sugiyama T., Gygi S., Grewal S.I., and Moazed D. 2004. RNAi-mediated targeting of heterochromatin by the RITS complex. Science 303: 672.

Volpe T.A., Kidner C., Hall I.M., Teng G., Grewal S.I., and Martienssen R.A. 2002. Regulation of heterochromatic silencing and histone H3 lysine-9 methylation by RNAi. Science 297: 1833.

West A.G., Gaszner M., and Felsenfeld G. 2002. Insulators: Many functions, many mechanisms. Genes Dev. 16: 271.

Whetstine J.R., Nottke A., Lan F., Huarte M., Smolikov S., Chen Z., Spooner E., Li E., Zhang G., Colaiacovo M., and Shi Y. 2006. Reversal of histone lysine trimethylation by the JMJD2 family of histone demethylases. Cell 125: 467.

Yamada T., Fischle W., Sugiyama T., Allis C.D., and Grewal S.I. 2005. The nucleation and maintenance of heterochromatin by a histone deacetylase in fission yeast. Mol. Cell 20: 173.

Yang W. and Steitz T.A. 1995. Recombining the structures of HIV integrase, RuvC and RNase H. Structure 3: 131.

Yuan Y.R., Pei Y., Ma J.B., Kuryavyi V., Zhadina M., Meister G., Chen H.Y., Dauter Z., Tuschl T., and Patel D.J. 2005. Crystal structure of $A$. aeolicus argonaute, a site-specific DNA-guided endoribonuclease, provides insights into RISCmediated mRNA cleavage. Mol. Cell 19: 405.

Zofall M. and Grewal S.I. 2006. Swi6/HP1 recruits a JmjC domain protein to facilitate transcription of heterochromatic repeats. Mol. Cell 22: 681 . 


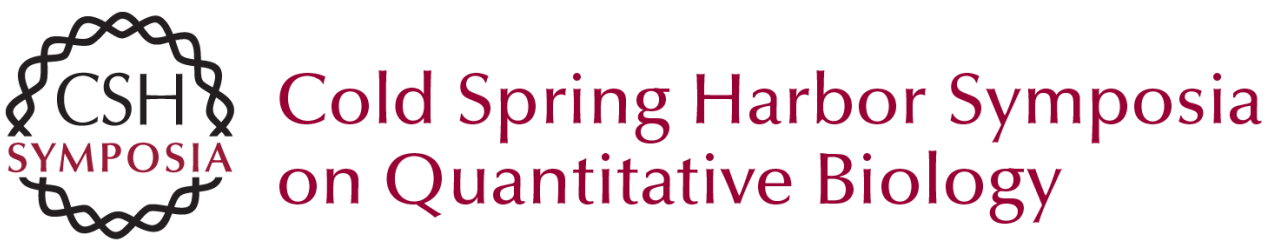

\section{RNAi-mediated Heterochromatin Assembly in Fission Yeast}

M. ZOFALL and S.I.S. GREWAL

Cold Spring Harb Symp Quant Biol 2006 71: 487-496

Access the most recent version at doi:10.1101/sqb.2006.71.059

References This article cites 95 articles, 44 of which can be accessed free at: http://symposium.cshlp.org/content/71/487.full.html\#ref-list-1

\section{License}

Email Alerting Receive free email alerts when new articles cite this article - sign up in Service the box at the top right corner of the article or click here. 